\title{
003 POSTTRAUMATIC STRESS DISORDER AND THE BRAIN
}

doi:10.1136/jnnp-2013-306103.3

Chris Brewin

Chris Brewin is a Consultant Clinical Psychologist at the Traumatic Stress Clinic and Professor in the Research Department of Clinical, Educational \& Health Psychology, University College London. He is a joint author of the dual representation theory of posttraumatic stress disorder, was centrally involved in shaping and overseeing Camden \& Islington's lead role in the mental health response to the 2005 London bombings, and is the author of "Posttraumatic Stress Disorder: Malady or myth?" (Yale University Press 2003).

A substantial number of structural and functional neuroimaging studies have been conducted with individuals suffering from PTSD. Many of them, however, have produced strikingly similar sets of findings to studies of depression and schizophrenia, for example involving reduced hippocampal volume and deficits in prefrontal control over limbic structures, particularly the amygdala. Investigations typically include only one psychiatric diagnosis and overlook the considerable symptom overlap between disorders. Also frequently overlooked is the presence of childhood adversity in patients' backgrounds, which is associated with neural changes in its own right. Hence previous research has not succeeded in distinguishing the correlates of generic aetiological or psychopathological factors from the specific processes that differentiate one disorder from another. This is essential if viable and well-targeted neurobiological models are to be developed that have clinical applications. Most imaging research has drawn on a limited set of basic science models such as fear conditioning. Despite its important contribution, the fear conditioning model of PTSD and paradigms such as script-driven imagery do not address important features such as the conscious reexperiencing of trauma and the distinction between voluntary and involuntary memory. The revised dual representation theory of PTSD (DRT) focusses on the difference between voluntary trauma memories and involuntary flashbacks, vivid images that are experienced in the present. Flashbacks are an important treatment target and are addressed in trauma-focussed cognitive-behaviour therapy. According to DRT, flashbacks are primarily supported by sensation-based representations created by activity in the dorsal visual stream, insula, and amygdala that have become disconnected from corresponding contextualised representations created by activity in the ventral visual stream and medial temporal lobe. Structural and functional studies will be described that focus on flashbacks and attempt to distinguish how the neural signature of PTSD is different from depression. Understanding the underlying processes and neural basis of these therapeutic target symptoms promises to generate more precise treatments, whether psychological or biological. 